\title{
Oroxylin A modulates mitochondrial function and apoptosis in human colon cancer cells by inducing mitochondrial translocation of wild-type p53
}

\author{
Chen Qiao ${ }^{1, *}$, Na Lu ${ }^{1, *}$, Yuxin Zhou ${ }^{1}$, Ting Ni ${ }^{1}$, Yuanyuan Dai ${ }^{1}$, Zhiyu Li ${ }^{2}$, Qinglong Guo ${ }^{1}$, \\ Libin Wei ${ }^{1}$ \\ ${ }^{1}$ State Key Laboratory of Natural Medicines, Jiangsu Key Laboratory of Carcinogenesis and Intervention, China Pharmaceutical \\ University, Nanjing 210009, People's Republic of China \\ ${ }^{2}$ Department of Medicinal Chemistry, China Pharmaceutical University, Nanjing 210009, People's Republic of China \\ *These authors contributed equally to this work
}

Correspondence to: Qinglong Guo, e-mail: anticancer_drug@163.com

Libin Wei, M.D, e-mail: wlbiws_1986@aliyun.com

Keywords: $p 53$, translocation, oroxylin A, oxidative stress

Received: August 11, 2015 Accepted: January 09, $2016 \quad$ Published: March 05, 2016

\section{ABSTRACT}

Oroxylin A is a flavonoid extracted from the root of Scutellaria baicalensis Georgi. We previously demonstrated that oroxylin A induced apoptosis in human colon cancer cells via the mitochondrial pathway. In the present study, we investigated the underlying mechanisms responsible for the mitochondrial apoptotic pathway triggered by oroxylin A. p53 regulates mitochondrial survival, mitochondrial DNA integrity, and protection from oxidative stress. We determined that oroxylin A induces p53 mitochondrial translocation and inhibits SOD2 activity. Additionally, our studies demonstrate that oroxylin A promotes the formation and mitochondrial translocation of the p53-Recql4 complex in HCT-116 cells. Finally, we showed that oroxylin A triggers cytosolic p53 activation, thereby promoting apoptosis. Mitochondrial translocation of p53 was also validated in vivo. Thus, oroxylin A induces mitochondrial translocation of p53 and leads to mitochondrial dysfunction in human colon cancer cells.

\section{INTRODUCTION}

P53 is one of the most well-characterized proteins in cancer cell biology and is widely recognized as a tumor suppressor [1]. The functions of p53 include regulation of substrate metabolism, DNA repair, autophagy, cell senescence, angiogenesis, and oxidative stress [2]. Although most p53-dependent cellular processes are nuclear-based [3, 4], mitochondrial translocation of p53 has recently been detected raising the possibility of organelle-based effects [5, 6]. Mitochondrial translocation of p53 can suppress manganese superoxide dismutase (MnSOD), an antioxidant defense enzyme, and induce intracellular accumulation of reactive oxygen species (ROS) [7]. P53 can also activate several mitochondrial apoptosis-related proteinsF such as $\mathrm{Bcl}-\mathrm{xL}$ to promote caspase activation and cytochrome $\mathrm{C}$ release [8].

The Recql4 protein is essential for p53 mitochondrial translocation and recruits $\mathrm{p} 53$ to the mitochondria in response to stress $[9,10]$. Localization of p53 to the mitochondria via a mitochondrial localization signal can induce mitochondrial DNA replication and contribute to changes in the mitochondrial physiological status [9]. Oroxylin $\mathrm{A}\left(\mathrm{C}_{16} \mathrm{H}_{12} \mathrm{O}_{5}\right)$ is a flavonoid isolated from the root of traditional herbal medicine Scutellaria baicalensis Georgi. Recently, oroxylin A has been shown to have anticancer activities in vitro and in vivo through multiple mechanisms including cell cycle arrest, inhibition of metastasis, and apoptosis [11-13]. In this study, we provide evidence that oroxylin A facilitates mitochondrial translocation of $\mathrm{p} 53$ thereby inducing the mitochondrial apoptotic pathway.

\section{RESULTS}

Translocation of p53 to the mitochondria inhibits SOD2 activity and promotes ROS generation in wt-p53 cancer cells

Wt-p53 rapidly translocated to mitochondria in response to apoptosis-induced stress signals (particularly ROS generation). To confirm wt-p53 translocation to the 
mitochondria in various cancer cells, we evaluated the subcellular distribution of p53 and ROS levels in wt-p53 cancer cells (e.g., HCT-116, MDA-MB-231, and HepG 2 cells), mut-p53 cancer cells (e.g., SW480, MCF-7), and normal cells (e.g., L02 cells) after TPA treatment [14]. After treatment of the cells with TPA for $24 \mathrm{~h}, \mathrm{p} 53$ translocated to the mitochondria in the wt-p53 cancer cells but not in mut-p53 cancer cells or normal cells (Figure 1A). The ROS level in wt-p53 cancer cells showed a corresponding increase and SOD2 activity decreased (Figure 1B-1D). These results demonstrated that wt-p53 translocation contributed to inhibition of mitochondrial
SOD2 activity and increased ROS levels in wt-p53 cancer cells.

\section{Oroxylin A induces apoptosis by promoting mitochondrial translocation of wt-p53 in HCT-116 cells}

We previously demonstrated that oroxylin A had therapeutic potential in human colon cancer cells via a ROS-related mitochondrial pathway [13] in which p53 was stabilized and glycolysis inhibited [15]. Here, we determined that oroxylin A treatment increased the levels of
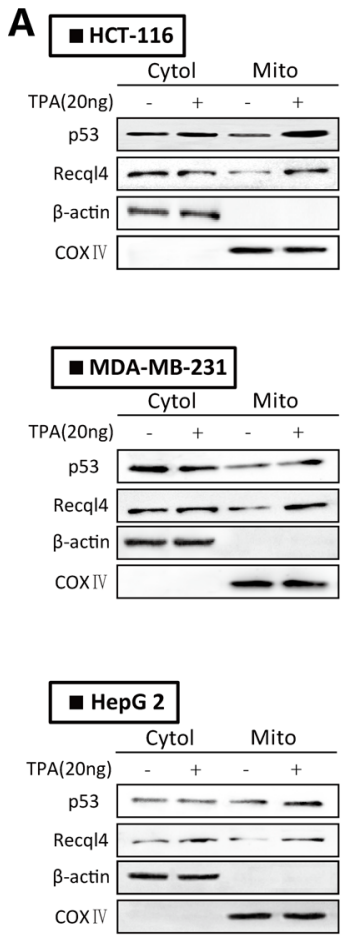

C

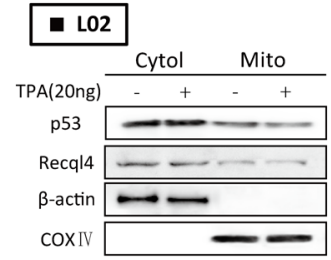

B

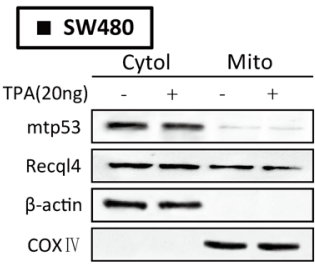

\section{MCF-7}

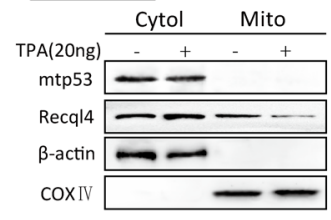
$-$

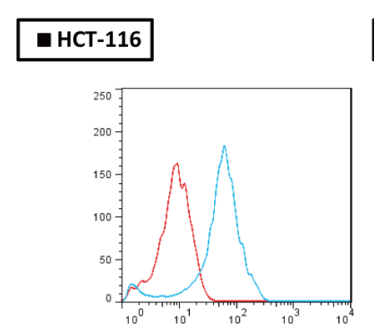

- MDA-MB-231
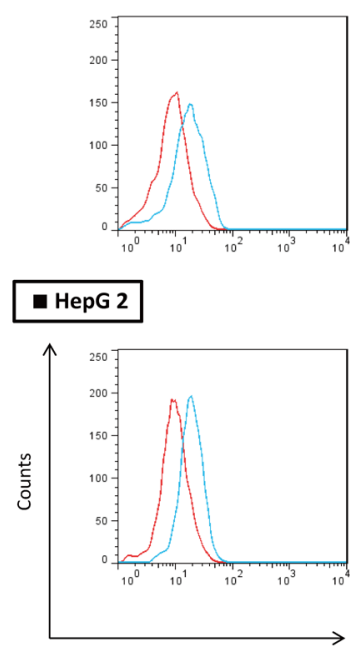

D

ROS
- SW480

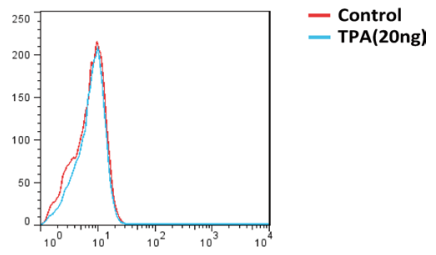

- MCF-7

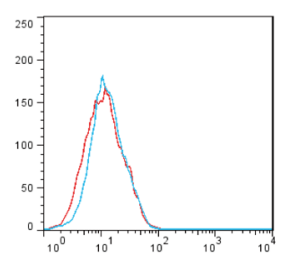

- LO2

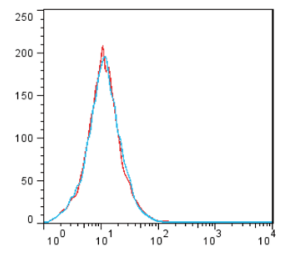

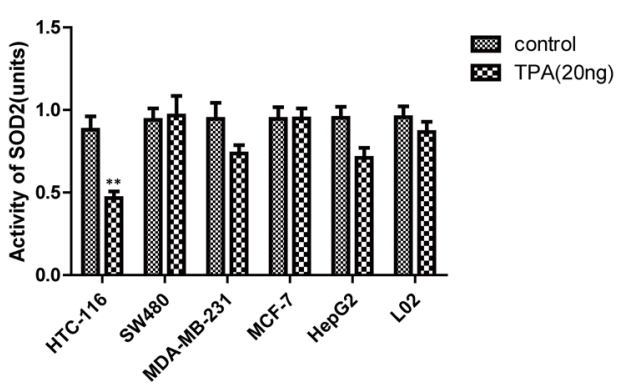

Figure 1: The accumulation of p53 in mitochondria leads to inhibition of SOD2 and increased ROS generation in wt-p53 cancer cells. (A) Western blotting analysis of mitochondrial p53. (B) ROS production was monitored using $10 \mu \mathrm{M}$ DCFH-DA and detected by flow cytometry. (C) Quantification of ROS levels. (D) Evaluation of SOD2 activity. Bar, SD. ${ }^{*} P<0.05$ or $* * P<0.01$ versus the untreated control. 
mitochondrial p53 in HCT-116 cells in a dosage-dependent manner. In contrast, mitochondrial translocation of p53 was not observed in mut-p53 SW480 cells (Figure 2A). Oroxylin A increased ROS levels and decreased SOD2 activity (Figure 2B-2D) in HCT-116 but not in SW480 cells (Figure 2E-2G). The growth of HCT-116 cells was inhibited by oroxylin A while the growth of SW480 cells did not change significantly over time (Figure 2H-2J). These results indicated that oroxylin A promoted mitochondrial accumulation of wt-p53 resulting in inhibition of mitochondrial SOD2 activity, increased ROS levels, and induction of apoptosis.

\section{Oroxylin A-induced inhibition of SOD2 activity and increased ROS were mediated by wt-p53 mitochondrial translocation in HCT-116 cells}

We next investigated the importance of p53 in the oroxylin A-induced effects by silencing p53 in HCT116 cells. The level of AcLys68 SOD2 reflected the activation status. Knockdown of p53 attenuated the inhibition of SOD2 activity by oroxylin A (Figure 3A-3F). The oroxylin A-induced increases in ROS levels and apoptosis were simultaneously attenuated. Recql4 is essential for p53 mitochondrial translocation [9]. Following Recq14 knockdown, p53 could not localize to mitochondria. Oroxylin-induced inhibition of SOD2 activity and increased ROS were also weakened (Figure 3G-3J). Total p53 levels did not significantly change (Figure $3 \mathrm{~K}$ ). We also treated HCT-116 cells with pifithrin- $\mu$, an inhibitor of p53 mitochondrial translocation, and found that the effects of oroxylin A were attenuated (Figure 3L-3O). Confocal imaging revealed that deletion of both Recql4 and pifithrin- $\mu$ inhibited oroxylin A-induced p53 mitochondrial translocation (Figure 3P-3Q). These results suggested that wt-p53 mitochondrial translocation had a key role in oroxylin A-induced inhibition of SOD2 activity and apoptosis in HCT-116 cells.

\section{P53 mutation inhibits oroxylin A-induced mitochondrial translocation}

To further characterize the key roles of p53 in oroxylin A-induced mitochondrial translocation, we transfected p53-null HCT-116 cells with either wt-p53 or mut-p53. When cells were transfected with wt-p53, oroxylin A treatment induced release of p53 from the nucleus and mitochondrial translocation. However, when
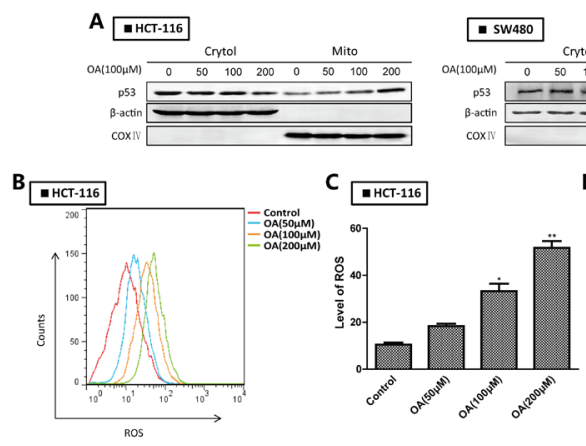

D $\mathbf{H C T}-116$
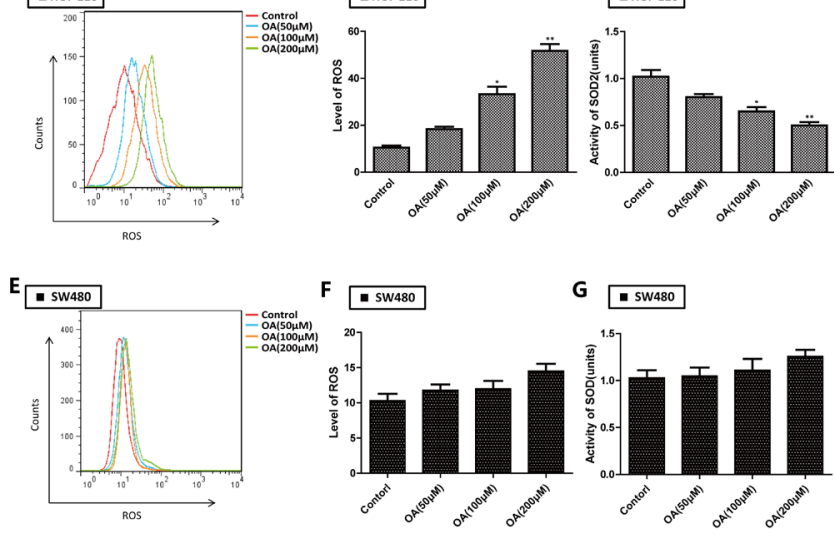

G

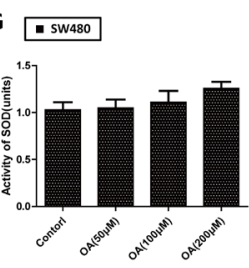

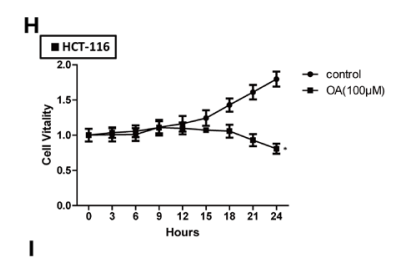
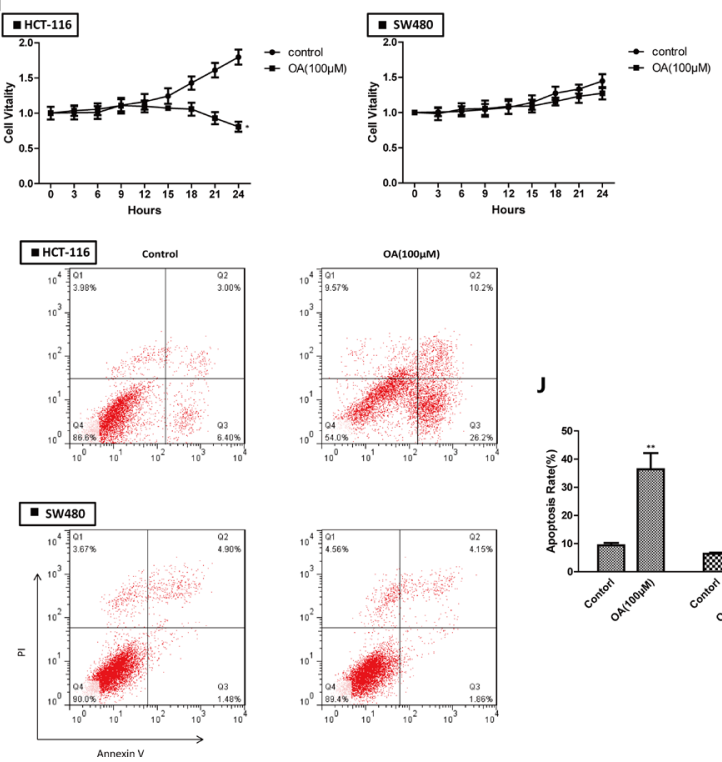

Figure 2: Oroxylin A induces apoptosis through p53 mitochondrial translocation in HCT-116 cells. (A) Western blotting analysis of p53 accumulation in mitochondria after oroxylin A treatment $(50 \mu \mathrm{M}, 100 \mu \mathrm{M}$, and $200 \mu \mathrm{M})$ for 24 h. (B-C) ROS production was monitored using $10 \mu \mathrm{M}$ DCFH-DA and then detected by flow cytometry after treatment of HCT-116 cells with $100 \mu \mathrm{M}$ oroxylin A for $24 \mathrm{~h}$. ROS levels were then quantified. Bar, SD. $* P<0.05$ or $* * P<0.01$ versus the untreated control. (D) The activity of SOD2 was evaluated as described above. (E-F) ROS production was monitored in SW480 after treatment of with $100 \mu \mathrm{M}$ oroxylin A for $24 \mathrm{~h}$ cells using $10 \mu \mathrm{M}$ DCFH-DA and then detected by flow cytometry. ROS levels were then quantified. Bar, SD. $* P<0.05$ or $* * P<0.01$ versus the untreated control. (G) The activity of SOD2 was evaluated as described above. (H) HCT-116 and SW480 cells were seeded in 96-well plates, incubated overnight, and treated with $100 \mu \mathrm{M}$ oroxylin A. The results of these experiments are shown as the mean \pm SD. (I-J) After treatment with $100 \mu \mathrm{M}$ oroxylin A for $24 \mathrm{~h}$, cells were stained with Annexin V and PI, and apoptotic cells detected by flow cytometry. Bar, SD. $* P<0.05$ or $* * P<0.01$ versus the untreated control. 

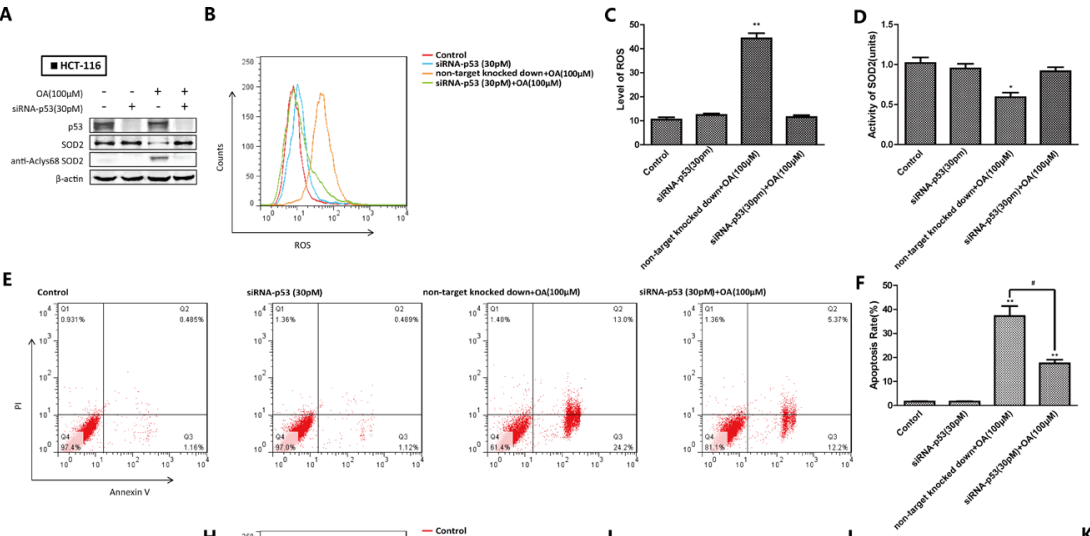
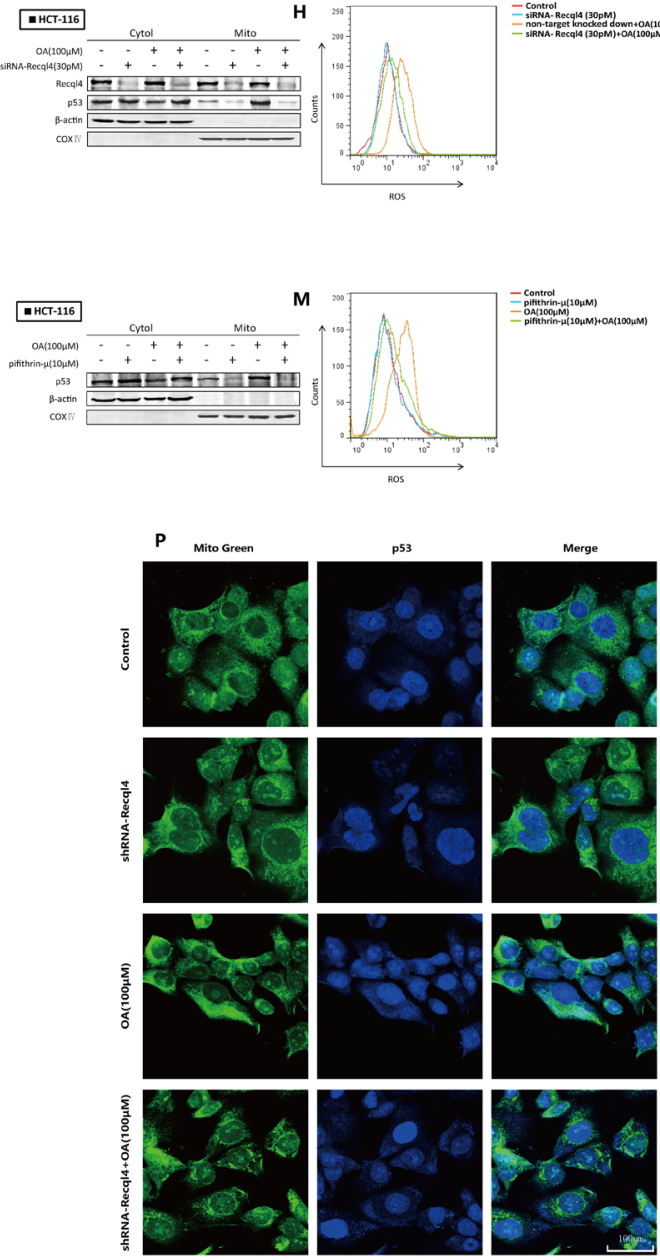
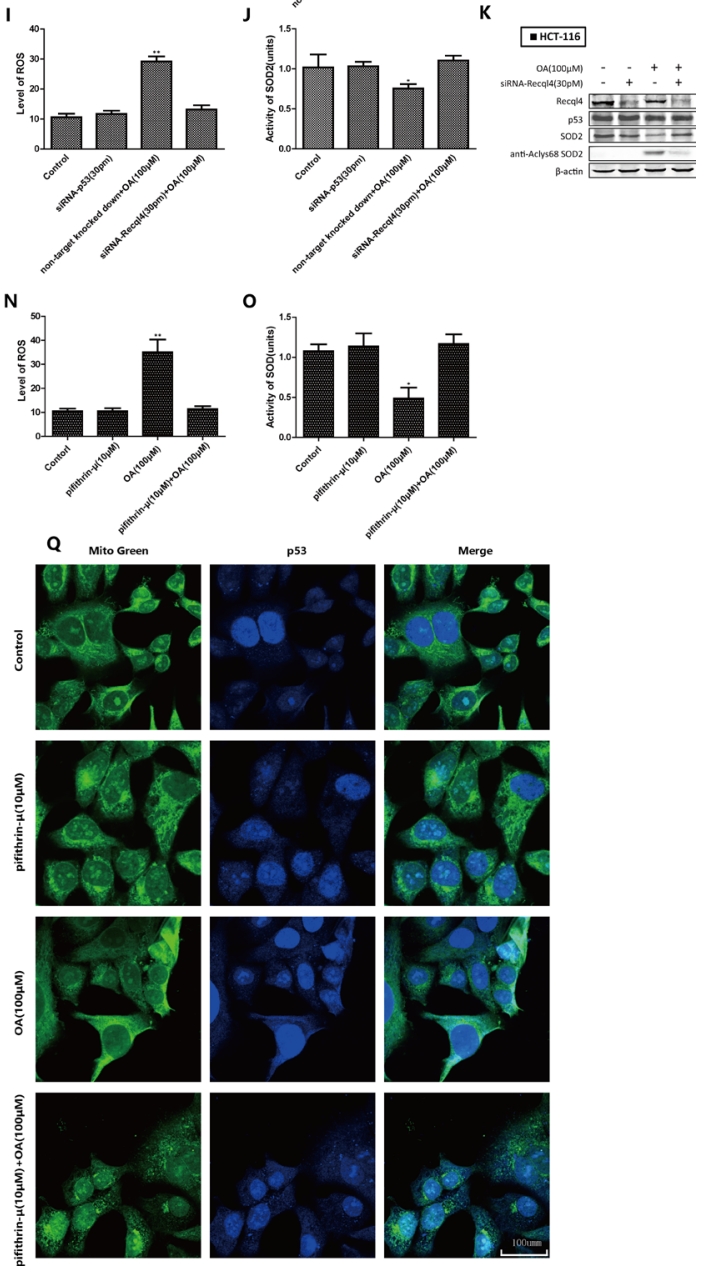

Figure 3: Oroxylin A-induced SOD2 activity inhibition and increased ROS were mediated by wt-p53 mitochondrial translocation in HCT-116 cells. (A) Western blot detection of SOD2 expression in p53 siRNA-transfected HCT-116 cells after treatment with $100 \mu \mathrm{M}$ oroxylin A for $24 \mathrm{~h}$. (B-C) Quantification of ROS production in p53 siRNA-transfected HCT-116 cells. (D) SOD2 activity in 533 siRNA-transfected HCT-116 cells. (E-F) Quantification of p53 siRNA-transfected HCT-116 apoptotic cells. (G) The subcellular distribution of p53 in Recq14 siRNA-transfected HCT-116 cells as detected by Western blot after treatment with $100 \mu \mathrm{M}$ oroxylin A for $24 \mathrm{~h}$. (H-I) Quantification of ROS production in Recq14 siRNA-transfected HCT-116 cells. (J) SO2 activity in Recq14 siRNA-transfected HCT-116 cells. (K) Western blot analysis of 53 expression in Recq14 siRNA-transfected HCT-116 cells after treatment with $100 \mu$ M oroxylin A for $24 \mathrm{~h}$. (L) HCT-116 cells were incubated with the indicated concentrations of DMSO, pifithrin- $\mu(10 \mu \mathrm{M})$, oroxylin A $(100 \mu \mathrm{M})$, and pifithrin- $\mu(10 \mu \mathrm{M})+$ oroxylin $\mathrm{A}(100 \mu \mathrm{M})$ for $24 \mathrm{~h}$, and the subcellular distribution of p53 analyzed by Western blotting. (M-N) ROS production in $\mathrm{p} 53$ pifithrin- $\mu(10 \mu \mathrm{M})$-treated HCT-116 cells was monitored as described above and ROS levels quantified. (O) SOD2 activity in pifithrin- $\mu(10 \mu \mathrm{M})$-treated HCT-116 cells was evaluated as described above. (P-Q) HCT-116 cells were incubated with the indicated concentrations of DMSO, shRNA/pifithrin- $\mu(10 \mu \mathrm{M})$, oroxylin A $(100 \mu \mathrm{M})$, and shRNA/pifithrin- $\mu(10 \mu \mathrm{M})+$ oroxylin A $(100 \mu \mathrm{M})$ for $24 \mathrm{~h}$. Confocal images of the cells show the fluorescence of p53 in blue, Mito in green, and the merged images in Column 3. Bar, SD. $* P<0.05$ or $* * P<0.01$ versus the untreated control. 
A
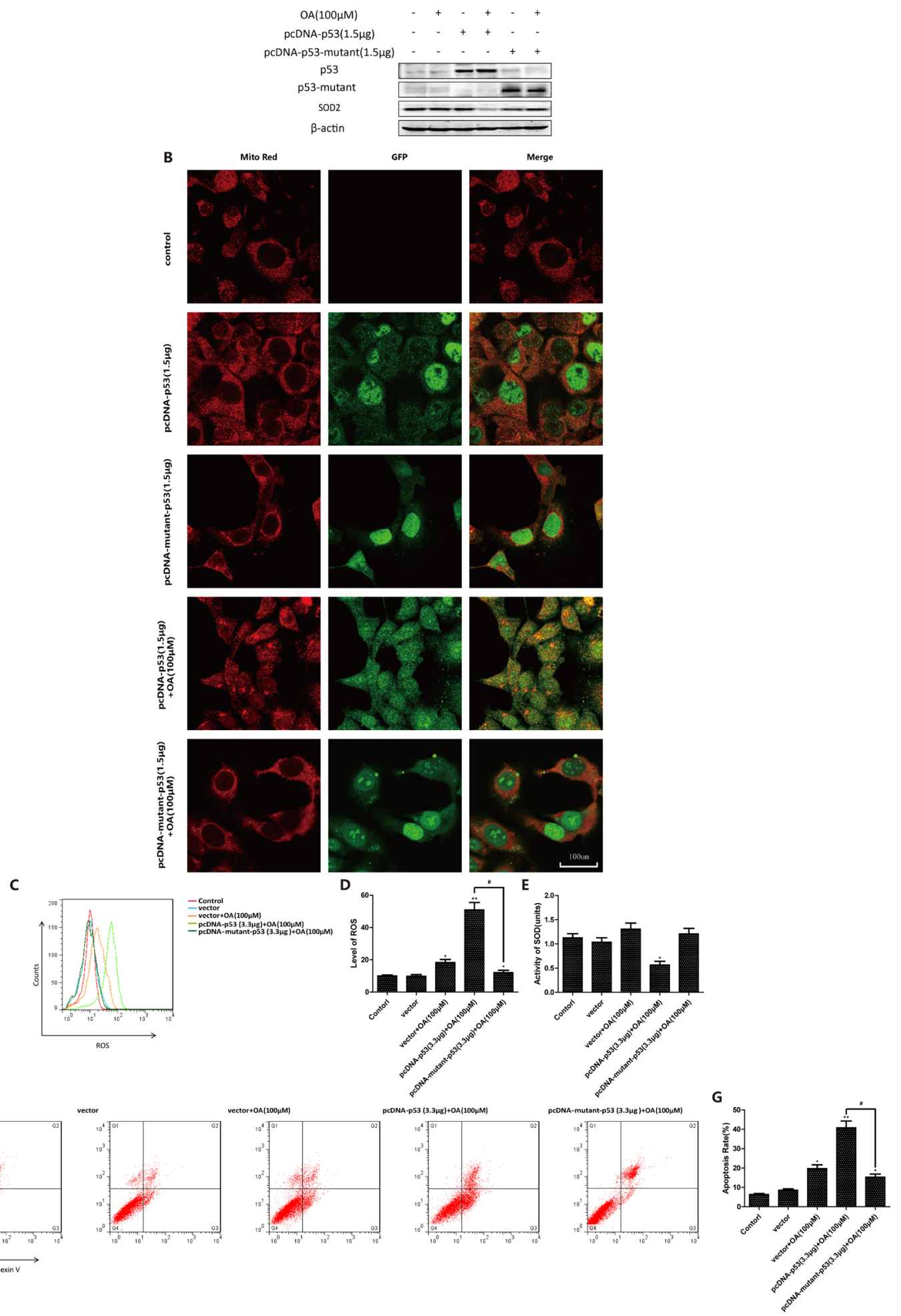

Figure 4: P53 mutation inhibits oroxylin A-induced mitochondrial translocation. (A) P53-null HCT-116 cells were incubated with the indicated concentrations of DMSO, pcDNA-p53 (1.5 $\mu \mathrm{g})$, oroxylin A $(100 \mu \mathrm{M})+$ pcDNA-p53 $(1.5 \mu \mathrm{g})$, pcDNA-mutant-p53 (1.5 $\mu \mathrm{g})$, or oroxylin $\mathrm{A}(100 \mu \mathrm{M})+$ pcDNA-mutant-p53 for $24 \mathrm{~h}$ and SOD2 expressin detected by Western blotting. (B) Confocal images of the cells show the fluorescence of Mito in red, p53/mutant-p53 in green, and the merged image in Column 3. (C-D) ROS production was analyzed after transfection and oroxylin A treatment $(100 \mu \mathrm{M})$ as described above. (E) SOD2 activity in p53/mutant-p53-transfected p53 null HCT-116 cells was evaluated as described above. (F-G) Apoptotic p53/mutant-p53 transfected p53-null HCT-116 cells were quantified as described above. Bar, SD. $* P<0.05$ or $* * P<0.01$ versus the untreated control. 
cells were transfected with mut-p53, p53 mitochondrial translocation was diminished (Figure 4A). In wt-p53transfected HCT116 cells, oroxoylin A decreased the levels of SOD2, increased ROS generation, and induced apoptosis. In contrast, in mut-p53 transfected HCT-116 cells, these parameters were not significantly altered compared to vector-transfected control cells (Figure 4B-4F). The changes in SOD2 activity showed the opposite pattern (Figure 4E). Moreover, oroxylin A alone promoted mitochondrial translocation of p53 in Caco-2 (p53-null) cells transfected with wt-p53. Decreased SOD2 activity and increased ROS levels as well as apoptosis were observed (Supplementary Figure 1). These results indicated that mitochondrial translocation of $\mathrm{p} 53$ could be prevented when p53 was mutated. Oroxoylin A could only promote the mitochondrial translocation of wt-p53.

\section{Oroxylin A induces mitochondrial translocation of the p53-Recql4 complex and cytosolic activation of p53 in HCT-116 cells}

As shown in Figure 5A, mitochondrial translocation of the p53-Recq14 complex was observed in response to oroxylin A treatment. Previous studies have indicated that cytosolic p53 can be captured by Bcl-xL and sequestered into an inactive complex in unstressed cells [16]. Therefore, we investigated complex formation between p53 and Bcl-xL in HCT-116 cells using immunoprecipitation. The binding capacity of p53 and Bcl-xL increased in TPA-treated cells but decreased in oroxylin A-treated cells (Figure 5B-5C). Bcl-2 can interact with p53 and neutralize its proapoptotic effects on Bax and Bak, which oligomerize and form lipid pores in the outer mitochondrial membrane [16]. Mitochondrial Bcl-2 increased significantly in TPA-treated cells but decreased in oroxylin A-treated cells (Figure 5D). These results demonstrated that oroxylin A induced p53 cytosolic activation and mitochondrial translocation in the presence of Bcl-xL and Bcl-2.

\section{Oroxylin A induces mitochondrial translocation of $\mathrm{p53}$ in vivo}

To verify the oroxylin A-induced mitochondrial translocation of p53 in vivo, we used an HCT-116 transplanted tumor model established in BALB/C nude mice. We injected $100 \mathrm{mg} / \mathrm{kg}$ oroxylin A intraperitoneally and sections of human tumor tissue were obtained and analyzed by immunohistochemistry after 21 days. The results indicated that both the volumes and weights of the transplanted tumors decreased after oroxylin A treatment, while the weights of the nude mice did not change (Figure 6A-6D). The inhibitory rate of tumor growth in the oroxylin A-treated group reached $50.80 \%$. Finally, wider distribution of p53 in the cytoplasm was observed by confocal microscopy compared to the control group (Figure 6E).

\section{DISCUSSION}

Mitochondrial translocation of p53 has been reported to have a direct impact on mitochondrial function, similar to the nuclear effects of p53 [17-20]. We previously demonstrated that oroxylin A can induce the mitochondrial apoptotic pathway [21-24]. We hypothesized that mitochondrial translocation of p53 might be the internal mechanism by which oroxylin A triggers mitochondria-mediated apoptosis. The subcellular distribution in various tumor cells after TPA treatment in HCT-116 cells demonstrated the highest sensitivity. Therefore, we selected HCT-116 cells for further research. Western blotting of mitochondrial extracts revealed that wt-p53 translocated to the mitochondria in response to oroxylin A treatment in HCT-116 cells but not in SW480 cells in which p53 was mutated. HCT-116 cells were also more vulnerable than SW480 cells in which the mechanisms of ROS resistance might be suppressed by oroxylin A-induced mitochondrial translocation of wt-p53.

We achieved the same results in the absence of Recq14 and after treatment with an inhibitor of p53 mitochondrial translocation, indicating that p53 exerts its pro-apoptotic effects in the mitochondria rather than in the cytoplasm after oroxylin A treatment. These results were validated in p53-null HCT-116 cell model and p53-null Caco-2 cells (Supplementary Figure 1). Confocal microscopy revealed that wt-p53 was released from the nucleus. Thus, the first step of p53 translocation was confirmed in oroxylin A-treated cells. The other two essential elements for the process of p53 mitochondrial translocation are cytosolic transport and activation [25]. Dual fluorescence staining demonstrated p53 and Recql4 colocalization in mitochondria, indicating that transport was activated normally in response to oroxylin A. TPA has the ability to drive these two processes. However, it triggered invasion while oroxylin A induced apoptosis [26]. There might be some differences in p53 activation in oroxylin A-treated cells compared to TPA-treated cells. Cytosolic p53 activity can be captured and neutralized by cytosolic Bcl-xL through formation of an inactive complex [27]. After TPA treatment, complex formation between Bcl-xL-p53 and p53-Recq14 increased, indicating initiation of a protective mechanism. After oroxylin A 

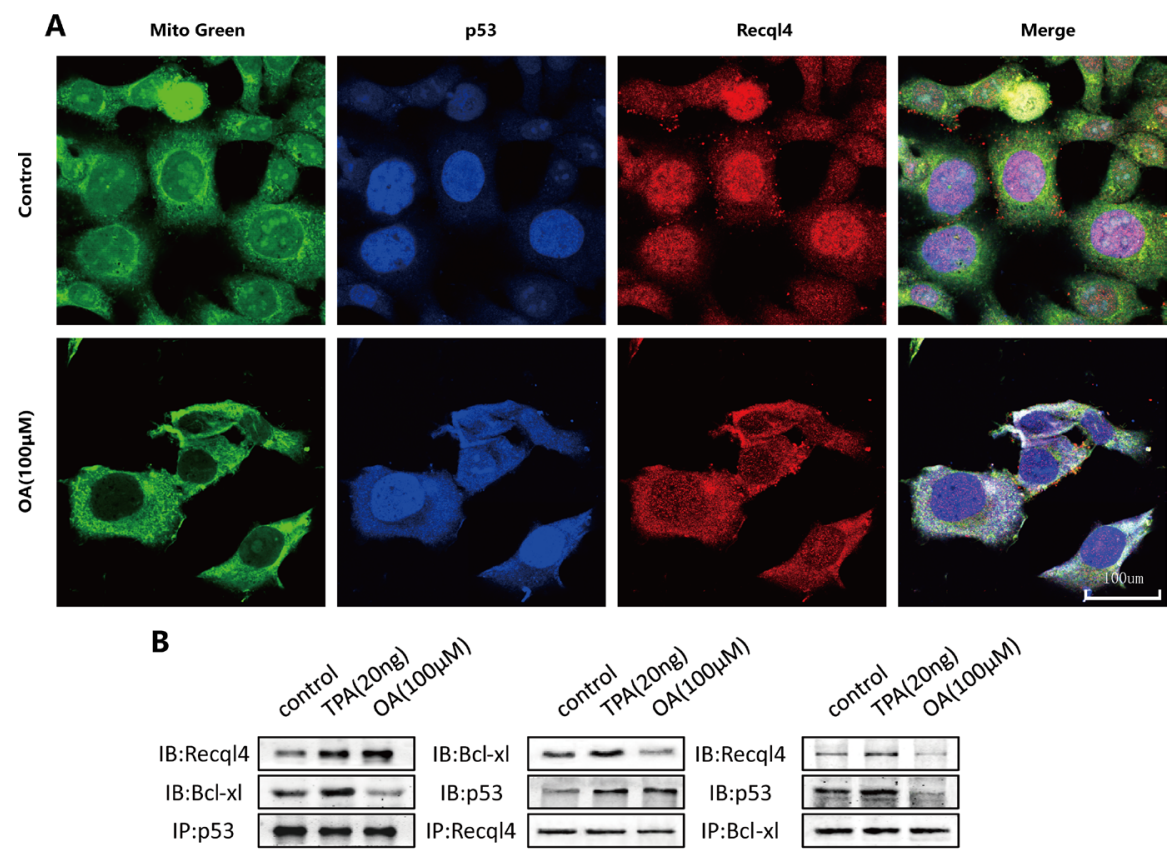

C

p53

Bcl-xl
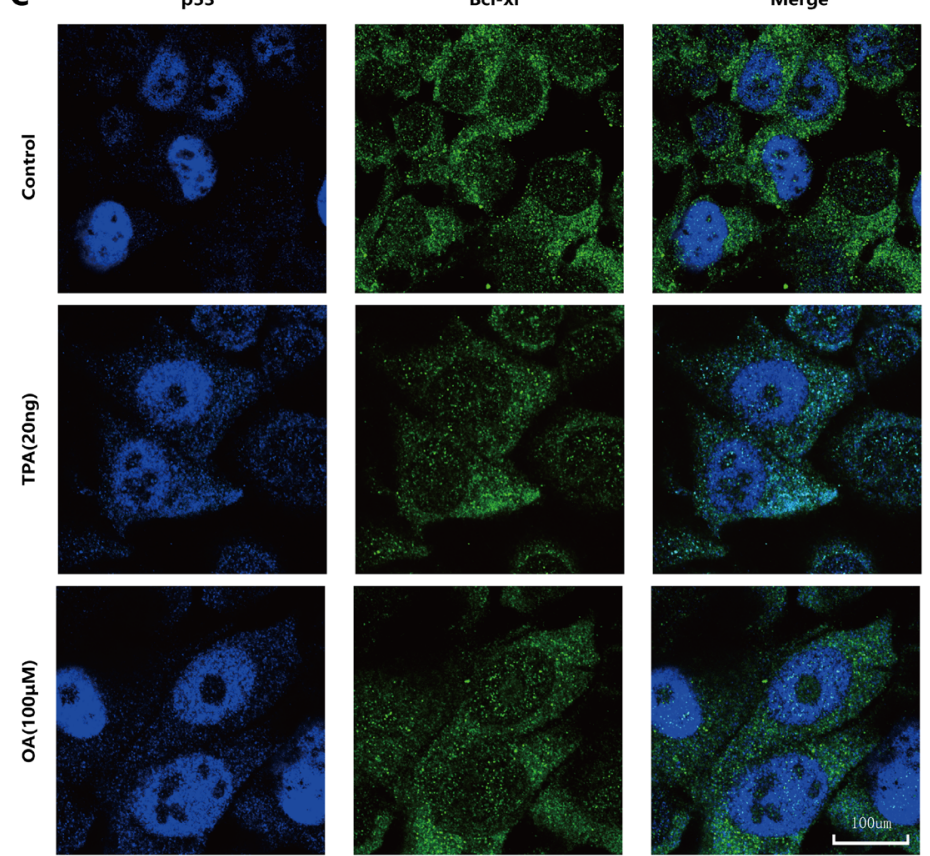

D

Mito

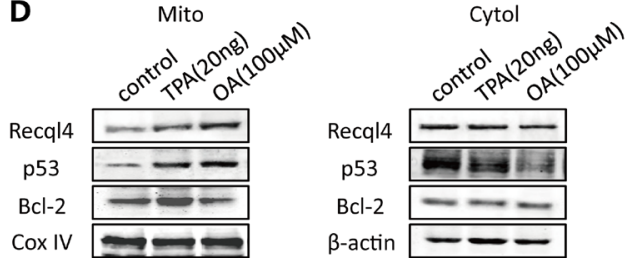

Figure 5: Oroxylin A induces cytosolic activation of p53 and recq14 mitochondrial translocation in HCT-116 cells. (A) HCT-116 cells were incubated with the indicated concentrations of DMSO or oroxylin A $(100 \mu \mathrm{M})$. Confocal images of the cells show the fluorescence of p53 in blue, Mito in green, Recq14 in red, and the merge in Column 4. (B) Antibodies to p53, Recq14, and Bcl-xL were used for the immunoprecipitation. Western blotting was used to detect complex formation between proteins. (C) HCT-116 cells were incubated with the indicated concentrations of DMSO, TPA $(20 \mathrm{ng})$, and oroxylin A (100 $\mu \mathrm{M})$. Confocal images of the cells show the fluorescence of 553 in blue, Bcl-xL in green, and the merged images in Column 3. (D) After the mitochondria were isolated from HCT-116 cells, Western blotting was performed to analyze Recq14, p53, and Bcl-2. 
treatment, binding of p53-Recq14 increased while Bcl-xL was liberated from the complex, allowing activated p53 to be transported. Finally, we verified oroxylin A-induced p53 translocation in vivo. As shown in Figure 5E, p53 exhibited a wider distribution in mitochondria.

In summary, our studies of HCT-116 colon cancer cells have shown that oroxylin A can induce the cytosolic activation and mitochondrial translocation of p53, which triggers the mitochondrial apoptotic pathway. We have elucidated the role of p53 mitochondrial translocation in oroxylin A-induced apoptosis, demonstrating that oroxylin A has the capacity to selectively inhibit tumor cells and lower toxicity. This could be applied to the development of anti-tumor drugs.
A

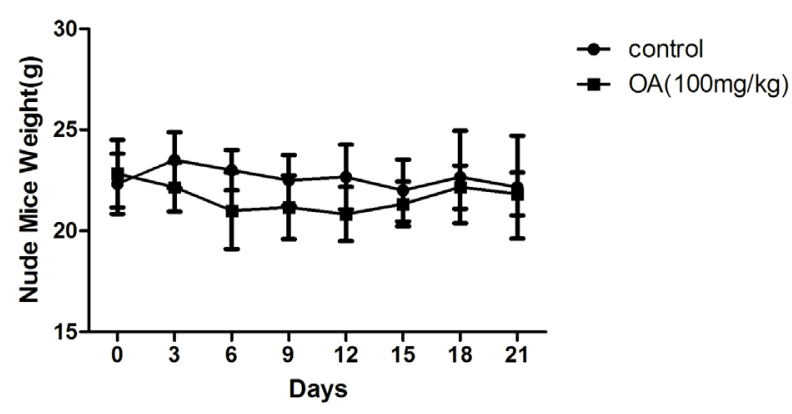

C

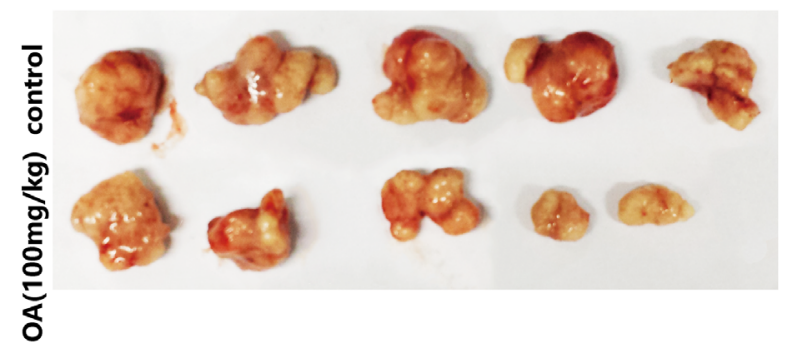

B

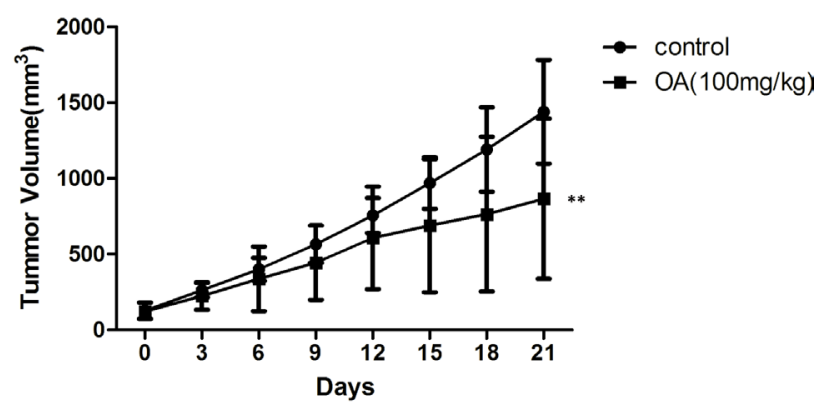

D

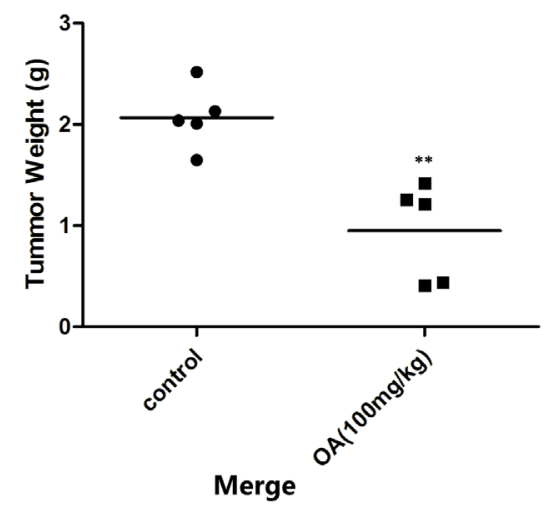

$\mathbf{E}$
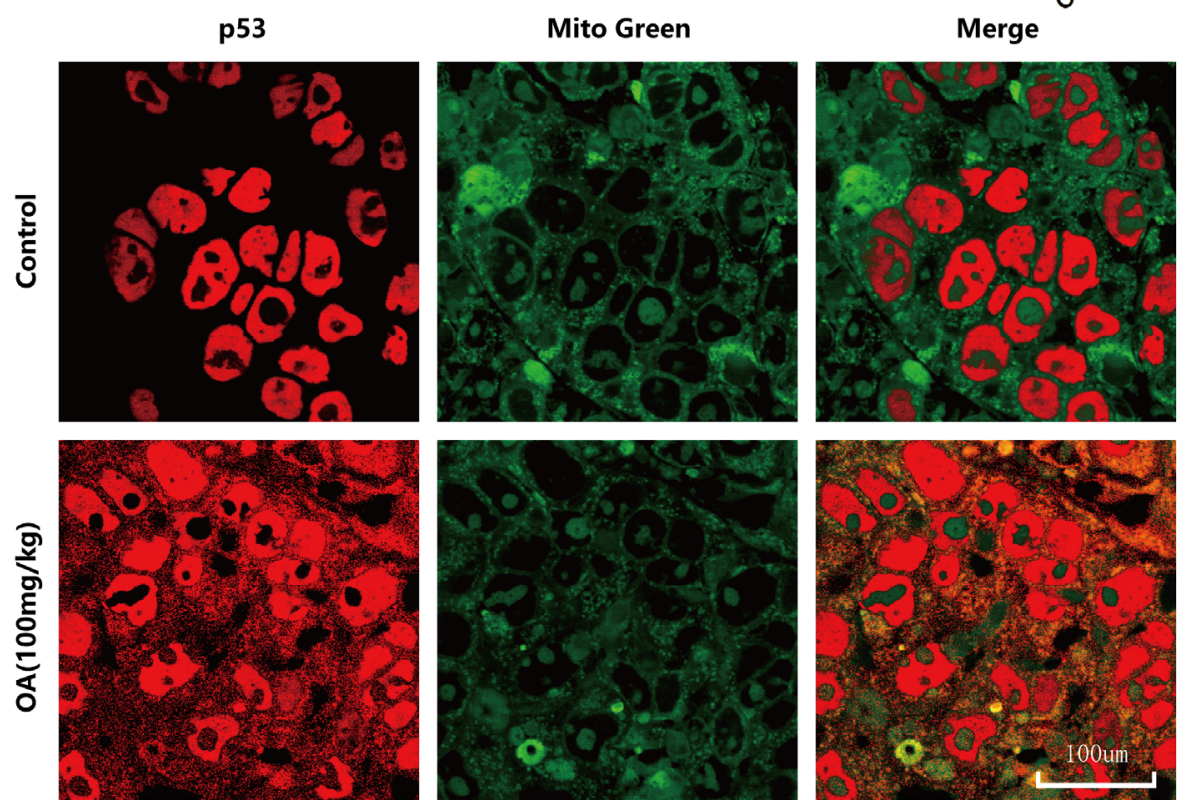

Figure 6: Oroxylin A induces Recql4-dependent mitochondrial translocation of p53 in vivo. (A-D) HCT-116 tumors were transplanted into BALB/C nude mice and $100 \mathrm{mg} / \mathrm{kg}$ of oroxylin A administrated by intraperitoneal injection. (E) Confocal images of the cells show the fluorescence of p53 in red, Mito in green, and the merged images in Column 3. 


\section{MATERIALS AND METHODS}

\section{Materials}

Oroxylin A was isolated from the root of Scutellaria baicalensis Georgi and dissolved in dimethyl sulfoxide (DMSO) according to previously published protocols. Samples that contained $\geq 99 \%$ oroxylin A were used in all experiments. Oroxylin A was dissolved in DMSO to $200 \mathrm{mM}$ and stored at $-20^{\circ} \mathrm{C}$. Before every experiment, a stock solution of oroxylin A was diluted in basal medium to various working concentrations. The 12-O-tetradecanoylphorbol 13-acetate (TPA) was purchased from Sigma-Aldrich (St. Louis, MO, USA) and dissolved in normal saline.

\section{Cell culture}

HCT-116, SW480, Caco-2, MDA-MB-231, MCF-7, HepG 2, and L02 cells were purchased from the Cell Bank of Shanghai Institute of Biochemistry and Cell Biology, Chinese Academy of Sciences (Shanghai, China). HCT-116 cells were maintained in 90\% McCoy's 5A medium (Sigma-Aldrich) supplemented with 10\% heat-inactivated fetal bovine serum (FBS) (GIBCO, Invitrogen, Carlsbad, CA, USA), $100 \mathrm{U} / \mathrm{mL}$ penicillin $\mathrm{G}$, and $100 \mu \mathrm{g} / \mathrm{mL}$ streptomycin. SW480, Caco-2, MDA-MB-231, and Hep G2 cells were maintained in 90\% Dulbecco's Modified Eagle Medium supplemented with $10 \%$ heat-inactivated FBS (Sijiqing, Hangzhou, China). MCF-7 cells and L02 cells were maintained in $90 \% 1640$ medium supplemented with $10 \%$ heat-inactivated FBS (Sijiqing, Hangzhou, China). The p53-null HCT116 cell line was provided by the Life Sciences Institute at the University of Michigan. All cell types described above were cultured at $37^{\circ} \mathrm{C}$ in a waterjacketed $\mathrm{CO}_{2}$ incubator (Thermo Fisher Scientific, Waltham, $\mathrm{MA}, \mathrm{USA}$ ) in a humidified atmosphere with $5 \% \mathrm{CO}_{2}$.

\section{Animal model}

Male athymic BALB/c nude mice (35-40 days old) with body weights ranging from 18-22 g were supplied by the Academy of Military Medical Sciences of the Chinese People's Liberation Army (Certificate No. SCXK-[Army] 2007-004). Animals were maintained at $22 \pm 2{ }^{\circ} \mathrm{C}$ with $55-65 \%$ humidity in stainless steel cages under controlled light (12 h light/day) and were fed with standard laboratory food and water. Animal care was provided in accordance with the Guide for the Care and Use of Laboratory Animals published by the United State National Institute of Health.

Twenty nude mice were inoculated subcutaneously with $1 \times 10^{7}$ HCT-116 cells into the right axilla. After 12 days of growth, tumor size was measured using micrometer calipers. Mice that were inoculated with
HCT-116 cells and had similar tumor volumes were randomly divided into the following two groups (five mice/group): saline control and oroxylin A (100 mg/kg intravenously every 2 days). Tumor size was measured every 3 days using micrometer calipers and tumor volume was calculated using the following formula: Tumor volume $\left(\mathrm{mm}^{3}\right)=\mathrm{d}^{2} \times \mathrm{D} / 2$, in which $\mathrm{d}$ and $\mathrm{D}$ were the shortest and the longest diameters, respectively. Mice were sacrificed on day 21 and tumor tissues analyzed by immunohistochemistry.

\section{Alamar blue assays}

HCT-116 and CaCo-2 cells were seeded in 96-well plates, incubated overnight, and the treated with $100 \mu \mathrm{M}$ oroxylin A. Alamar blue assays (Invitrogen) were then performed according to the manufacturer's instructions. Fluorescence was analyzed once an hour for $9 \mathrm{~h}$ at an excitation wavelength of $530-560 \mathrm{~nm}$ and an emission wavelength of $590 \mathrm{~nm}$ using a fluorospectrophotometer (Olympus, Japan). The Alamar blue assay incorporates a fluorometric/colorimetric growth indicator based on detection of metabolic activity. Specifically, the system incorporates an oxidationreduction indicator that both fluoresces and changes color in response to chemical reduction of the growth medium that results from cell growth.

\section{Western blotting}

Proteins were isolated using lysis buffer, incubated in SDS buffer, separated by SDS-PAGE, and transferred onto PVDF membranes. Antibodies to Bcl-2 (sc-7382), p53 (sc-6243), Recq14 (sc-366840), and SOD2 (sc-133134) were purchased from Santa Cruz Biotechnology (Santa Cruz, CA, USA). The antibody to Bcl-xL (2764p) was purchased from Cell Signaling Technology (Danvers, MA, USA). Antibodies to Cox IV (ab14744) and SOD2 acetylated at Lysine 68 (AcLys68) (ab137037) were purchased from Abcam (Hong Kong, China), and the antibody to $\beta$-actin (BM0627) was purchased from Boster Biotechnology (Wuhan, China).

\section{Annexin V/propidium iodide staining}

Apoptotic cells were identified using Annexin V-FITC Apoptosis Detection Kits (KeyGen, Naijing, China) according to the manufacturer's instructions. In brief, $1 \times 10^{6}$ cells were harvested, washed, and suspended in PBS. Cells were then resuspended in $500 \mu \mathrm{L}$ Binding Buffer, and $5 \mu \mathrm{L}$ AnnexinV-FITC and $5 \mu \mathrm{L}$ propidium iodide were added. Apoptotic cell death was analyzed using FACSCalibur flow cytometry (Becton Dickinson, San Jose, CA, USA) immediately after double supravital staining. 


\section{Measurement of intracellular ROS}

Intracellular ROS was detected by DCFH-DA (Beyotime Institute of Biotechnology, Haimen, China). Cells were harvested from 6-well plates, washed with PBS, resuspended in serum-free medium, and then incubated with $10 \mu \mathrm{M}$ DCFH-DA for $30 \mathrm{~min}$ at $37^{\circ} \mathrm{C}$ in the dark. The fluorescence intensity $(488 \mathrm{~nm}$ excitation and $525 \mathrm{~nm}$ emission) was measured immediately using FACSCalibur flow cytometry (Becton Dickinson).

\section{SOD2 activity assays}

SOD2 activity was assayed using the $\mathrm{Cu} / \mathrm{Zn}$ SOD and Mn-SOD Assay Kit (KeyGen) using the manufacturer's protocols. Briefly, cells were first collected and lysed, and then $3 \mathrm{mM}$ potassium cyanide was added to the lysate to inhibit both $\mathrm{Cu} / \mathrm{Zn}-\mathrm{SOD}$ and extracellular SOD, which allowed detection of Mn-SOD activity. Samples were assayed in the absence of xanthine oxidase to generate a background sample. After the samples and SOD standards were prepared and added to a 96-well plate, the reaction was initiated by adding $20 \mu \mathrm{L}$ of diluted xanthine oxidase to all wells. The plate was then incubated on a shaker for $30 \mathrm{~min}$ at room temperature. Optical density values were detected using a spectrophotometer (Thermo Fisher Scientific) at $450 \mathrm{~nm}$.

SOD activity was quantified using the following equation obtained from the linear regression of the standard curve substituting the linearized rate (LR, LR $\left.=\left(\mathrm{A}_{\text {blank } 1}-\mathrm{A}_{\text {blank } 2}-\mathrm{A}_{\text {sample }}\right) /\left(\mathrm{A}_{\text {blank1 } 1}-\mathrm{A}_{\text {blank } 2}\right) \times 100 \%\right)$ for each sample. One unit was defined as the amount of enzyme that exhibited $50 \%$ dismutation of the superoxide radical. Thus, SOD activity sample $(\mathrm{U})=\mathrm{LR}_{\text {sample }} /\left(1-\mathrm{LR}_{\text {sample }}\right)$ units.

\section{Transient transfection of plasmids and siRNA}

The pcDNA3-p53-GFP and pcDNA3-p53-mutantGFP plasmids were obtained from Addgene (Addgene 11770; Addgene 24859, Addgene Cambridge, MA, USA). The siRNAs for p53 and Recql4 were purchased from Santa Cruz Biotechnology. For transfection, HCT-116 cells were seeded in 6-well plates at $70 \%$ confluency, and then siRNA-p53 (30 pM)/siRNA-Recq14 (30 pM) were introduced into the cells using Lipofectamine 2000 (Invitrogen) according to the manufacturer's instructions. P53 null HCT-116 cells and Caco-2 cells were incubated with pcDNA-p53-GFP/pcDNA-p53-mutant-GFP and transfected using the same method. The cells were then exposed to oroxylin A or vehicle and harvested for further analysis.

\section{Stable transfection of shRNA}

The Recql4 shRNA plasmid (TL309882) was obtained from Origene (Rockville, MA, USA). HEK293 cells were seeded at a density of $3 \times 10^{5}$ cells in $2 \mathrm{~mL}$ into one well of a 6-well plate. The cells were then transfected with the Recql4 shRNA plasmid using Lipofectamine 2000 (Invitrogen) according to the manufacturer's recommendations Selection pressure was maintained by the presence of approximately $0.5-1.0 \mu \mathrm{g} / \mathrm{mL}$ puromycin. After the packaged cells reached approximately $70-80 \%$ confluency, the culture media was collected and passed through a $0.45 \mu \mathrm{m}$ filter. The virus stock and $4 \mu \mathrm{g} / \mathrm{mL}$ polybrene in growth medium were then added directly to the target cells.

\section{Immunofluorescence}

Cells were rinsed with PBS and fixed with 4\% paraformaldehyde in phosphate buffer ( $\mathrm{pH} 7.4$ ) for $20 \mathrm{~min}$ at room temperature. After washing with PBST, the cells were blocked with $3 \%$ bovine serum albumin in PBST for $1 \mathrm{~h}$, incubated with anti-p53 antibody/anti-Recq14 antibody (Santa Cruz Biotechnology) overnight at $4^{\circ} \mathrm{C}$, and then washed with PBST. Tetramethylrhodamine-labeled antimouse/anti-rabbit IgG antibody (Rockland, Limerick, PA, USA) was then added and the cells incubated for $1 \mathrm{~h}$. Mitochondria were identified by Mito Red (KeyGen) according to the manufacturer's instructions. Finally, the cells were rinsed with PBST and exposed to DAPI for $15 \mathrm{~min}$ to stain the nuclei. After washing with PBS, the cells were examined under a laser scanning confocal microscope FV10-ASW [Ver 2.1] (Olympus Corp, MPE FV1000) for co-localization analysis.

\section{Tissue immunofluorescence}

Tissue sections (4 $\mathrm{mm}$ thick) were dewaxed and rehydrated through graded washes of alcohol in distilled water $(100,95,85,75 \%)$. The sections were then boiled in citrate buffer at $98^{\circ} \mathrm{C}$ for antigen retrieval and treated with $3 \%$ hydrogen peroxide to block endogenous peroxidase activity. Finally, the sections were incubated with a protein-blocking agent (kit 9710 MAIXIN, MaixinBio Co., Fuzhou, Fujian) prior to the application of the primary antibody. Slides were incubated with the anti-p53 antibody (Santa Cruz Biotechnology) overnight at $4{ }^{\circ} \mathrm{C}$ and then washed with PBST. Mitochondria were identified using Mito Red (KeyGen) according to the manufacturer's protocol. After washing with PBS, tissues were examined under a laser scanning confocal microscope FV10-ASW [Ver 2.1] (Olympus Corp, MPE FV1000).

\section{Immunoprecipitation}

Recaq14, Bcl-xL, and p53 were immunoprecipitated from the total protein using polyclonal antibodies crosslinked to protein G-agarose beads (Santa Cruz Biotechnology). The immune complexes were analyzed by Western blotting and probed with antibodies against Bcl-xL, p53 of interest. 


\section{Preparation of mitochondria- and cytosol- enriched extracts}

Following the $48 \mathrm{~h}$ incubation of the cells with oroxylin A, mitochondria/cytosolic fractionation was then performed using the Mitochondria/Cytosol Fractionation Kit (BioViso, VA, USA) according to the manufacturer's protocol.

\section{Statistical analysis}

The data are presented as the mean \pm standard deviation (SD) from triplicate parallel experiments unless otherwise indicated. Statistical analyses were performed using one-way ANOVA.

\section{ACKNOWLEDGMENTS AND FUNDING}

This work was supported by the National Science \& Technology Major Project (No. 2012ZX09304-001, 2012ZX09103101-050, 2013ZX09103-001-007), Program for Changjiang Scholars and Innovative Research Team in University (IRT1193), Project Program of the State Key Laboratory of Natural Medicines, China Pharmaceutical University (No. SKLNMZZJQ201302, No. G140042), National Natural Science Foundation of China (No. 81373448, 81503097), Science Foundation for Distinguished Young Scholars of Jiangsu Province (BK20150028), and Program for New Century Excellent Talents in University (NCET-12-0973).

\section{CONFLICTS OF INTREST}

None.

\section{REFERENCES}

1. Bartlett JD, Morton JP. p53: the tumour suppressor turns mitochondrial regulator. J Physiol. 2013; 591:3455-3456.

2. Vousden KH, Lane DP. p53 in health and disease. Nat Rev Mol Cell Biol. 2007; 8:275-283.

3. Bourdon JC, Deguin-Chambon V, Lelong JC, Dessen P, May P, Debuire B, May E. Further characterisation of the p53 responsive element - identification of new candidate genes for trans-activation by p53. Oncogene. 1997; 14:85-94.

4. Polyak K, Xia Y, Zweier JL, Kinzler KW, Vogelstein B. A model for p53-induced apoptosis. Nature. 1997; 389:300-305.

5. Donahue RJ, Razmara M, Hoek JB, Knudsen TB. Direct influence of the p53 tumor suppressor on mitochondrial biogenesis and function. FASEB J. 2001; 15:635-644.

6. Dhar SK, St Clair DK. Nucleophosmin blocks mitochondrial localization of p53 and apoptosis. J Biol Chem. 2009; 284:16409-16418.
7. Liu J, St Clair DK, Gu X, Zhao Y. Blocking mitochondrial permeability transition prevents p53 mitochondrial translocation during skin tumor promotion. FEBS Lett. 2008; 582:1319-1324.

8. Erster S, Mihara M, Kim RH, Petrenko O, Moll UM. In vivo mitochondrial p53 translocation triggers a rapid first wave of cell death in response to DNA damage that can precede p53 target gene activation. Mol Cell Biol. 2004; 24:6728-6741.

9. De S, Kumari J, Mudgal R, Modi P, Gupta S, Futami K, Goto H, Lindor NM, Furuichi Y, Mohanty D, Sengupta S. RECQL4 is essential for the transport of p53 to mitochondria in normal human cells in the absence of exogenous stress. J Cell Sci. 2012; 125:2509-2522.

10. Gupta S, De S, Srivastava V, Hussain M, Kumari J, Muniyappa K, Sengupta S. RECQL4 and p53 potentiate the activity of polymerase gamma and maintain the integrity of the human mitochondrial genome. Carcinogenesis. 2014; $35: 34-45$.

11. Liu W, Mu R, Nie FF, Yang Y, Wang J, Dai QS, Lu N, Qi Q, Rong JJ, Hu R, Wang XT, You QD, Guo QL. MAC-related mitochondrial pathway in oroxylin-A-induced apoptosis in human hepatocellular carcinoma HepG2 cells. Cancer Lett. 2009; 284:198-207.

12. Wei L, Dai Q, Zhou Y, Zou M, Li Z, Lu N, Guo Q. Oroxylin A sensitizes non-small cell lung cancer cells to anoikis via glucose-deprivation-like mechanisms: c-Src and hexokinase II. Biochim Biophys Acta. 2013; 1830: $3835-3845$.

13. Qiao C, Wei L, Dai Q, Zhou Y, Yin Q, Li Z, Xiao Y, Guo Q, $\mathrm{Lu}$ N. UCP2-related mitochondrial pathway participates in oroxylin A-induced apoptosis in human colon cancer cells. J Cell Physiol. 2015; 230:1054-1063.

14. Robbins D, Ponville J, Morris K, Zhao Y. Involvement of PTEN in TPA-mediated p53-activation in mouse skin epidermal JB6 cells. FEBS Lett. 2012; 586:4108-4113.

15. Zhao K, Zhou Y, Qiao C, Ni T, Li Z, Wang X, Guo Q, Lu N Wei L. Oroxylin A promotes PTEN-mediated negative regulation of MDM2 transcription via SIRT3-mediated deacetylation to stabilize p53 and inhibit glycolysis in wtp53 cancer cells. J Hematol Oncol. 2015; 8:41.

16. Vaseva AV, Moll UM. The mitochondrial p53 pathway. Biochim Biophys Acta. 2009; 1787:414-420.

17. Bensaad K, Tsuruta A, Selak MA, Vidal MN, Nakano K, Bartrons R, Gottlieb E, Vousden KH. TIGAR, a p53inducible regulator of glycolysis and apoptosis. Cell. 2006; 126:107-120.

18. Maddocks OD, Vousden KH. Metabolic regulation by p53. J Mol Med (Berl). 2011; 89:237-245.

19. Lee SY, Shin SJ, Kim HS. ERK1/2 activation mediated by the nutlin3induced mitochondrial translocation of $\mathrm{p} 53$. Int $\mathrm{J}$ Oncol. 2013; 42:1027-1035. 
20. Xavier JM, Morgado AL, Sola S, Rodrigues CM. Mitochondrial translocation of p53 modulates neuronal fate by preventing differentiation-induced mitochondrial stress. Antioxid Redox Signal. 2014; 21:1009-1024.

21. Singh J, Kakkar P. Oroxylin A, a constituent of Oroxylum indicum inhibits adipogenesis and induces apoptosis in 3T3L1 cells. Phytomedicine. 2014; 21:1733-1741.

22. Zou M, Hu C, You Q, Zhang A, Wang X, Guo Q. Oroxylin A induces autophagy in human malignant glioma cells via the mTOR-STAT3-Notch signaling pathway. Mol Carcinog. 2014.

23. Wei L, Zhou Y, Qiao C, Ni T, Li Z, You Q, Guo Q, Lu N. Oroxylin A inhibits glycolysis-dependent proliferation of human breast cancer via promoting SIRT3-mediated SOD2 transcription and HIF1alpha destabilization. Cell Death Dis. 2015; 6:e1714.

24. Dai Q, Yin Y, Liu W, Wei L, Zhou Y, Li Z, You Q, Lu N, Guo Q. Two p53-related metabolic regulators, TIGAR and SCO2, contribute to oroxylin A-mediated glucose metabolism in human hepatoma HepG2 cells. Int J Biochem Cell Biol. 2013; 45:1468-1478.
25. Khatun S, Chaube SK, Bhattacharyya CN. p53 activation and mitochondria-mediated pathway are involved during hanging death-induced neuronal cell apoptosis in dentate gyrus region of the rat brain. Springerplus. 2013; 2:407.

26. Yen HR, Liu CJ, Yeh CC. Naringenin suppresses TPAinduced tumor invasion by suppressing multiple signal transduction pathways in human hepatocellular carcinoma cells. Chem Biol Interact. 2015; 235:1-9.

27. Sot B, Freund SM, Fersht AR. Comparative biophysical characterization of $\mathrm{p} 53$ with the pro-apoptotic BAK and the anti-apoptotic BCL-xL. J Biol Chem. 2007; 282: 29193-29200. 